\title{
Efficient Video Distribution over WiMAX-Enabled Networks for Healthcare and Video Surveillance Applications
}

\author{
Dmitry V. Tsitserov and Dmitry K. Zvikhachevsky \\ Lancaster University, \\ School of Computing and Communications,
}

UK

\section{Introduction}

In this chapter we present an efficient video distribution technique which is equally applicable to both E-health and surveillance applications running over IEEE802.16/WiMAX technology platform. The developed scheme contributes to resolving of ever-struggling challenge of optimal bandwidth allocation between competitive data-consuming applications in wireless communications. The introduced approach for combined utilization of WIMAX QoS guarantee mechanism with object/quality-segmented video streams enables to achieve an improved level of system performance when compared with conventional distribution algorithms. The test scenarios were verified through NS-2 computer simulations, whereas the obtained results report better model system behavior estimated in QoS metrics, such as per flow, summary throughputs, an average end-to-end delay, particularly evaluated as bandwidth utility gain.

The whole chapter consists of two sections which are structurally common, but focused on the specific application area. The first section is devoted to WiMAX consideration for Ehealth applications, while the second one addresses the same issues regarded video surveillance. Each section highlights important technical aspects of the communication technology which is well-suited for the relevant applications. There is also a brief review of up-to-date related research initiatives that are built on the existent standards like IEEE802.16/WiMAX and IEEE802.11/WiFi in each section. The detailed description of the experimental models, covering the suggested distribution technique, the case-study scenarios with simulation settings and appropriate results are separately accommodated in the according sub-sections. Finally, the chapter ends with the consequent conclusions.

\section{Efficient video distribution in E-health systems via WiMAX technology}

\subsection{E-health environment and diligent communication platform}

Recent technological breakthrough in wireless communications have extended the boundaries and enlarged the scope of the application fields that vividly contribute to human safety and healthcare. 
E (electronic)-health terminology lumps a variety of medicine and communication services associated with rendering of healthcare practice and delivering it to patients. The existent range of e-health definitions, including health care providers, consumer health informatics, health knowledge management, electronic health records, first response service e.t.c only discover how broad and purpose-specific the e-health sphere turns out to be. With development of new technologies E-health have been following and implementing these state-of the arts for advanced care services, such as from conventional PC archive records to the video conferencing suggested for online surgery monitoring. The obvious commonplace of the outlined contemporary innovations is to enhance efficiency of healthcare, improve reliability and facilitate service acceptability throughout a patient-GP/medical specialisthospital communication chain (Zvikhachevskaya, 2010). In order to support efficient delivery of healthcare and neighboring services to the consumers, a profound and cuttingedge telecommunication technology has to be opted for. Proper selection of the desired transport technology should be based on aggregation of the application-driven factors that conform to the advanced information systems applied in E-health, user-accessibility and comfort, flexible scalability and to be upgrade-appreciated. There are some healthcare services and its relevant technical applications that are presented in Table 1.1. (Zvikhachevskaya, 2010).

\begin{tabular}{|c|c|}
\hline Technical application & Healthcare services Example(s) \\
\hline Video conferencing & $\begin{array}{c}\text { - Virtual multi-disciplinary team meeting in Cancer Care } \\
\text { - Support for Minor Injury Units } \\
\text { - Training and supervision } \\
\cdot \text { Prison to hospital }\end{array}$ \\
\hline $\begin{array}{l}\text { Remote monitoring of } \\
\text { physiological or daily living } \\
\text { signs (real time or } \\
\text { asynchronously) }\end{array}$ & $\begin{array}{l}\text { • Falls monitoring } \\
\text { - Physiological monitoring of chronic COPD and Heart } \\
\text { Failure }(\mathrm{CHF}) \text { at home }\end{array}$ \\
\hline Virtual visiting & $\begin{array}{l}\text { - Remote supervision of home dialysis } \\
\text { - Nurse visits to terminally ill patients }\end{array}$ \\
\hline $\begin{array}{l}\text { Store and forward referrals } \\
\text { (for example sending history } \\
\text { plus images for expert } \\
\text { opinion) }\end{array}$ & - Teledermatology \\
\hline $\begin{array}{l}\text { Web access to own health } \\
\text { records and guidance }\end{array}$ & - HealthSpace \\
\hline Telephone and Call-centres & $\begin{array}{l}\text { • Tele consultation } \\
\text { - Reminders for medication and appointments }\end{array}$ \\
\hline
\end{tabular}

Table 1.1. Examples of the e-Health Technologies (Zvikhachevskaya, 2010 )

As it follows from the examples, provided in the Table 1.1, an adequate E-health infrastructure with a diversified service range should rely on telecommunication technology which accommodates a number of dominant properties not limited to:

- Resource availability. In healthcare-related services, the timely and errorless data distribution is crucial since human life and safety might be at stake. Due to the 
complicated nature of application-dependant traffic, such as multimedia for video conferencing, emergency video from first response ambulance and call-centre voice transfer, fair and sufficient resource allocation is inherently challenging, in particular, when system bandwidth is shared by multiple services within the same network.

- High date rate. Interactive sessions like on-line consultancy together with video conference facilities require high data rate support.

- Flexible QoS support. An effective QoS provisioning in E-health networks is expected to classify traffic and delegate relevant system budget in line with given priority. (Zvikhachevskaya, 2010). Priority might be set for specific categories of patients, data flows, medical services. For example in (Zvikhachevskaya, 2010; Skinner et al., 2006; Bobadilla et al., 2007), the 2 priority-level approach is introduced for on-line and off-line clinical activities. On-line application type includes multimedia connections of audio and video exchange, biomedical signals and vital parameters (such as ECG signal, blood pressure, oxygen saturation, etc.) transmission. Of-line type specifies clinical routine accesses to databases, queries to medical report database. Triple urgency model is presented in (Hu \& Kumar, 2003), in which the patients calls, that sensor-based telemedicine network covers, are referred to one out of 3 levels of urgency. The first level involves ambulance and emergency calls and is given the highest priority with rate-guaranteed and delay-bounded service parameters. The second level faces calls from seriously ill patients in needs of urgent information exchange. Finally, calls from wrist-worn sensors, detecting regular body conditions of the observed patients are treated with Best-Effort service provision. In addition to prioritized treatment, relevant QoS parameters of delay, rate variations, packet dropping rate and others are to be sturdily considered while performing resource allocation between demanding medical applications.

- Wireless and portability support. Wireless connectivity allows to cover rural destinations and remote WLANs (wireless local area network) frequently employed in small offices and medical departments. This also targets patients unable to regular visit clinics and conduct medical consultancy in hospitals located distantly. Wireless technology enables comfortable accessibility of on-line medical communication through active usage of portable mobile devices like smart-phones, I-pods, laptops that are in use by almost everyone. With progressive growth of portable wireless communication gadgets flooding the wireless market, these devices may potentially serve as a first-aid mini point which is able to rapidly connect you to your GP and get you adequately advised on medicine prescription regardless of your destination and activity. Moreover, based on GPS data support, integrated in most mobile phones, the immediate ambulance help may be delivered, if required.

- Mobility Support. Mobile communications bring forward important benefits for both the e-health end-users and the medical services and staff. Ambulance, equipped with a required mobile communication unit, is capable of immediate data transfer for an urgent call initiating with a basic response center, while moving along. The patients under observation with a mobile device in use are again in state of fast 2-way communication to prevent hazardous effects (Zvikhachevskaya, 2010). In healthcare services the failure to timely react might yield distressing results. Mobility factor enhances efficiency of treatment decision-making, patients care and makes e-health services more comfortable and accessible. 
- IP-compatible platform. IP supported transport technology allows to be successfully interfaced with multitude of information systems and properly integrated into the hybrid network architecture with easy access to Web domains and public LANs whatever data path medium they counts on.

Therefore, a justified healthcare service delivery may by based on the broadband wireless standards, such as WiFi, LTE-Advanced, WiMAX, 3G/GPRS that present broadband wireless connectivity with WLANs as well as can act as fast-speed wireless transport communication platform (WiMAX, LTE-Adv, 3G, GPRS). Having observed the outlined above, it is important to note that an utmost wireless technology is not consistent to completely substitute wired communications and technologies yet, due to the restricted coverage, limited channel capacity and the available wired global infrastructure, the Ehealth network is a part of. The wireless segments of the global E-health network, however, can be on par with alternative wired paths, scaling from backhaul transmission to last-mile and broadband WLAN access solutions.

An example of how possible E-health services can be delivered across wireless broadband connection nodes is presented in Fig.1.1. (Zvikhachevskaya, 2010)

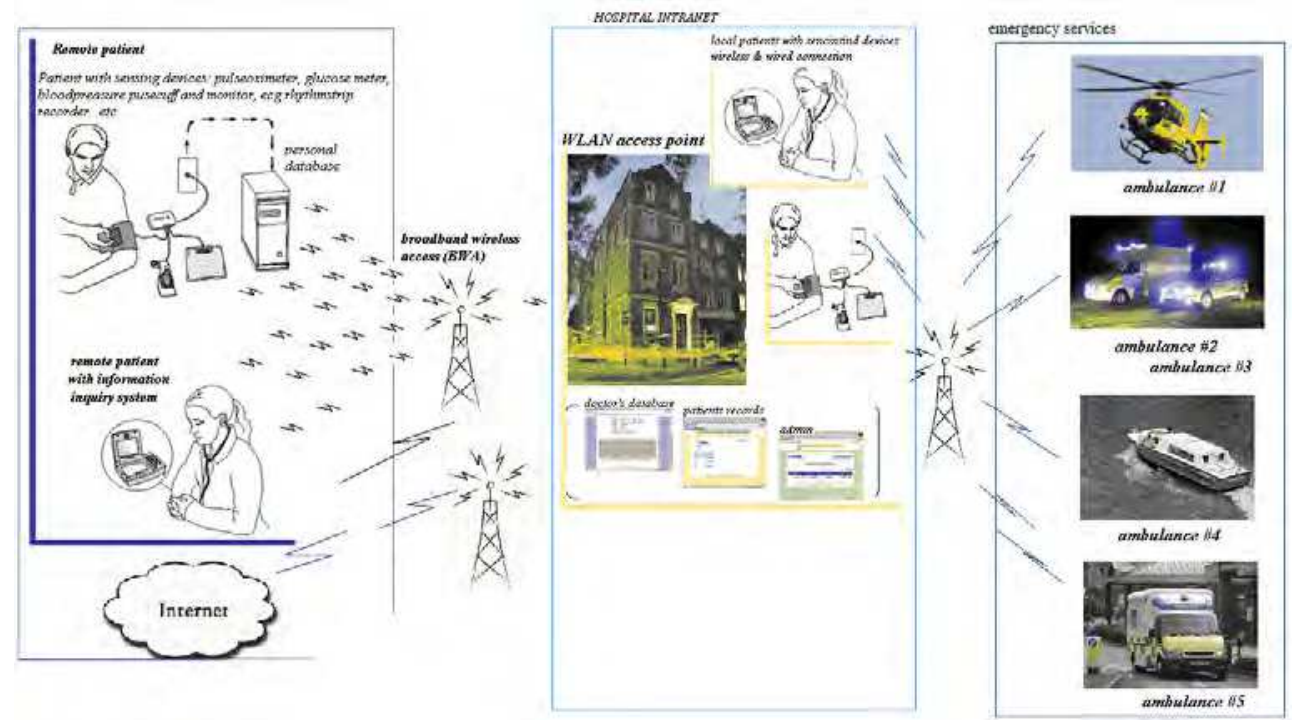

Fig. 1.1. The topology of E-health network and the participated users. (Zvikhachevskaya, 2010)

In this figure emergency services from multiple ambulances together with ordinary healthcare data of remote patients enter a hospital LAN through WBA (wirelses broadband access). Two-way communication is organized between the hospital centre and the involved users. The variety of core factors, such as a user remote distance, required traffic consumed, channel capacity, user moving speed, QoS guarantee and others will dominate the decision behind a suitable wireless system or combination of those. 


\subsection{Research advantages in E-health wireless communications}

There have recently been exposed research initiatives aimed at investigating of E-health system models within a wireless-supported framework. In (Y. Lin, et al., 2004) a mobile monitoring system is introduced to regular record patient's medical parameters like heartrate, three-lead electrocardiography through accommodation of PDA (personal digital assistant ) at a patient side and hospital WLAN technologies respectively. The objective of research carried out by Kutar and outlined in (Hu \& Kumar, 2006) is to assess telemedicine wireless sensor network behavior on the ground of 3G technology. An energy-efficient query resolution tool is examined when a guaranteed QoS mechanism for arriving multimedia calls is required in a large-scale network topology. Mobile WAP phone communication is proposed in (Maglaveras, et al., 2002) to maintain interactive data exchange among a generic contact centre and remote patients. The promising outcome justifies such an implementation, specifically siutable for applications of the chronic disease type. Much research efforts were focused on exploration of reliable and feasible QoS means to support quality-distinctive traffic distribution in the context of versatile telemedicine services. Due to multiple telemedicine scenarios, the involved services are aggregated into a single healthcare network that should secure a certain level of performance to data streams, the particular users, associated with the relevant applications. For example, real-time IPTV, VoIP data are delay-sensitive and data rate-guarantee considered and it is always a QoSrelated issue when network capacity is bounded with insufficient resources. Handy traffic management, therefore, is of great importance for E-health service provisioning. Addressing this problem, (Hu \& Kumar, 2003) have examined the use of energy-efficient query resolution mechanism for QoS-relied handling of arriving multimedia calls within a mobile wireless sensor network proposed for 3-G telemedicine applications. QoS consideration for wireless video transfer over ATM connections in medical environment was observed in (Dudzik, et al., 2009). In this review, ATM-based architecture allows ensure low delay and high bandwidth demands in mobile video services which positively impact on treatment efficiency of distant patients. IEEE 802.11 standard for WLAN connectivity was thoroughly explored for the purpose of its utilization across e-health mobile applications. Although, the standard is incapable of suiting real-time video and voice traffic demands on account of no priority provision and lack of service differentiation between various data flows, there is a great deal of research activity targeting QoS-accumulated techniques to maintain a certain level of QoS assurance in healthcare services. (Vergados et. al, 2006) Vergados pushes forward a challenge by proposing (Differentiated Services) wireless network architecture to support some e-Health applications with different QoS constraints. The developed DiffServ architecture is designed for emergency e-Health service and incorporates QoS mechanism that gets medical data transmission appropriately linked to different classes of service. The used resource allocation scheme considers urgency hierarchy of each application and its service-oriented QoS boundaries. The performance evaluation proves the obvious advantages of the proposed architecture in mobile telemedicine.

Yi Liu in (Y. Liu, et al., 2006) studies the emerging IEEE 802.11e standard for Wireless Local Area Networks (WLANs) with emphasis made on incoming data admission policy. In this QoS strategy, channel access parameters (CAPs) are assigned to different access categories (ACs). An admission control scheme is exploited to get the wireless system resources ultimately consumed in such a way, that let the upcoming real time traffic enter the network whilst leaving the existent data connections within the agreed QoS characteristics. The novel 
admission and congestion control scheme, introduced in the paper, performs regular analysis of traffic QoS requirements to assess admission control parameters for further updating the CAPs with help of adaptive channel conditions feedback. The extensive simulation of the proposed scheme demonstrates viability of guaranteed QoS mechanisms for real-time traffic in terms of guaranteed throughput indications, restricted delay and maximum dropping rate under efficient resource utilization.

D.Gao and J.Cai in (Gao \&Cai, 2005) have given a broad overview of the cutting edge admission control techniques for QoS-supported traffic management across the evolving IEEE 802.11e-enabled WLANs. This survey faces the research outcomes that have highlighted both EDCA and HCCA admission control schemes. It has been shown in this manuscript how utilization of the novel MAC QoS-related elaborations in EDCA and HCCA allow for telemedicine multimedia applications to be well considered in the quality and data admission control context of WLANs.

IEEE 802.16 or WiMAX standard also provides a great deal of efficient properties which make its utilization attractive across telemedicine application scenarios.

In contrast to IEEE802.11 standards suite, IEEE 802.16 is able to cover more spacious areas (over $50 \mathrm{~km}$ in radius against 150-200m achieved with WiFI) with higher data rates of up to $72 \mathrm{MBpsec}$ in optimal conditions. In addition, the diversified and powerful QoS-supported platform adopted in WiMAX allows handling numerous data types in conformance with specific telemedicine applications service demands, what is relatively limited for wireless Ehealth networks with WiFi-enabled assess technology (Noimanee, 2010).

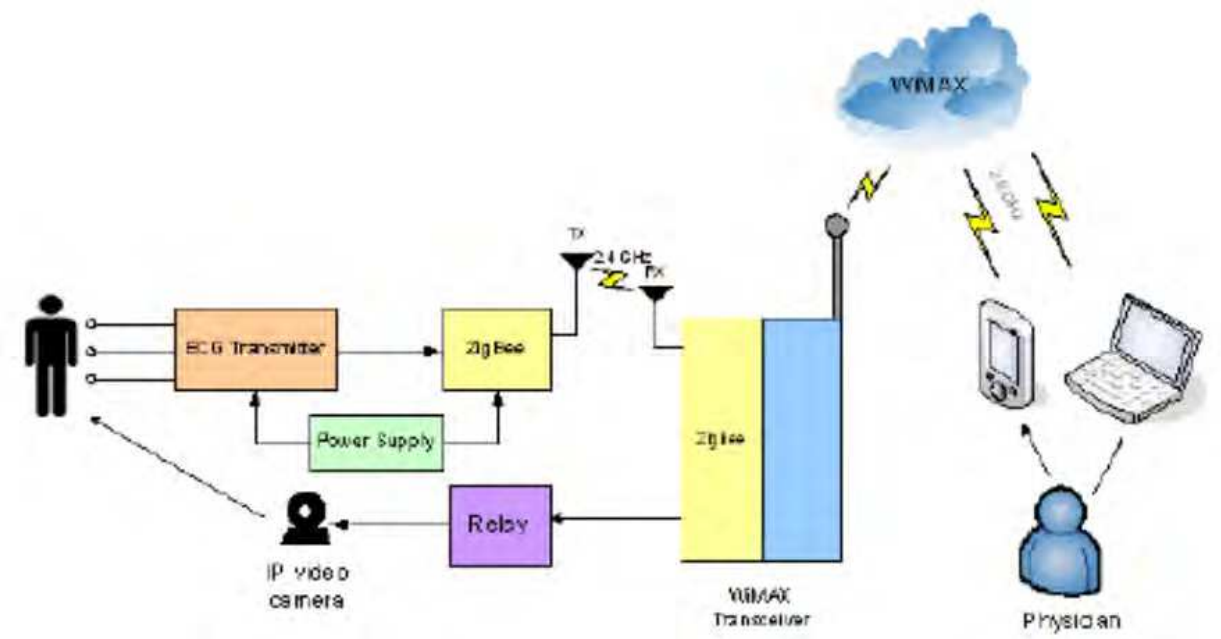

Fig. 1.2. The structure of on-line consult-based medical WiMAX system.

Considering that, WiMAX attracts intensive E-health practical and computer system modeling tailored to a particular telemedicine scenario. Thus, in (Noimanee, 2010) the authors designed and tested the global architecture for on-line monitoring and consultancy of remote patients with heart-related abnormal functionality detected through ECG signal measurement. The proposed solution enables for remote patients to regular send ECG 
signals measured on portable wrist-worn devices through the ZigBee/IEEE RF module to the responsible physicians through a WiMAX transceiver. In case of abnormal symptoms, the medical staff is able to remotely monitor relevant patients on application-run PDA or a wireless Laptop by activating the nearby IP surveillance camera via WiMAX connections.

The structure of the proposed system is shown on Figure 1.2 and encompasses 4 main subsegments, in particular:

1. ECG transceiver equipped with ZigBee module for sending ECG signals.

2. IP camera for panning patient video.

3. WiMAX access point allows delivering patient video and ECG signals to physicians.

4. Physicians personal equipment to view panning video and perform data analysis which supports medical consult-based services.

The highlighted WiMAX-based telemedicine system have demonstrated much satisfaction on delivering monitoring and consultancy services through wireless communication channels in the course of real experiments with engaged factory equipment. WiMAX technology proves to be efficient means for fast and easy data transfer, video monitoring and effective patient-physician collaboration.

In our investigation we also adhere to IEEE802.16 technology for its fine suitability to the general E-health network essentials, namely: high data rate together with long distance coverage, IP compatibility with co-existing neighboring network paths, prioritized treatment of different traffic types and QoS management, mobility support. In many examples of E-Health services local area connections are not sufficient. IEEE 802.16/WiMAX technology can eliminate these drawbacks by providing broadband connectivity over existing networks for $\mathrm{m}$-Health both fixed and mobile $\mathrm{m}$-Health users in a wireless metropolitan area network environment. In addition, IEEE802.16 standard is one of the emerging candidates for the next generation of International Mobile Telecommunications (IMT) - advanced systems. This facilitates further modernization and scalable integration of previously installed WiMAX systems into on-going AMT-Advanced network framework. Therefore, we select IEEE 802.16e standard as a baseline specification for our simulations.

We propose a novel algorithm for video distribution over IEEE 802.16 networks for mHealth applications. We assume that the proposed technique will operate over existing wireless broadband systems installed in hospitals or any of $\mathrm{m}$-Health dedicated environments. Therefore, there is a need for accommodating additional m-Health related traffic over existing networks. The proposed technique also allows utilization of the valueadded services with intensive bandwidth requirements.

This work is based on our previous research (Tsitserov et. al, 2008; Markarian et. al, 2010) which is concerned with the distribution of object-oriented MPEG streams over WiMAX network with exploitation of service flows embedded in WiMAX specifications. In this paper we analyze bandwidth resource allocation depending on a scheduling algorithm and apply splitting of video traffic to evaluate system critical states. Based on the developed software model we optimize the process of video data segmentation and verify the developed technique through case study scenarios, such as E-Health applications.

In case studies, various QoS-dependant streams were emulated to quantify the achievable improvement in the overall network throughput and identify the critical issues that 
influence the performance. As it follows from the experimental results, the proposed segmentation of real-time data flows provides both quantitative and qualitative system resources utilization. In the next subsection the developed performance model for segmented distribution of medical video data and discussions on advantages and issues of using WiMAX technology for E-Health applications are described. Further on, the developed scheduling algorithm together with simulation parameters and results are presented. In conclusion, test results and open problems are summarized and discussed.

\subsection{Distribution framework and simulation model}

\section{A Service mapping}

The QoS concept incorporated in the IEEE802.16 standard assumes the ability to manage incoming traffic based on application requirements. Although the set of functionalities and recommendations specified for QoS support in WiMAX are conceptually approved, the scheduling design and explicit structure is left up to vendors and research bodies for further development and implementation (J. G. Andrews, 2007). In the rest of this paper we will explore these areas and apply our results for efficient video distribution over WiMAX networks, ensuring full compatibility with existing and emerging standard specifications.

Users of fixed and mobile E-Health applications can access services via IEEE 802.16/WiMAX technology. Hence, owing to the guaranteed large bandwidth available, it can help to considerably reduce the transmission delay, for e.g. of video and high resolution ultrasound and radiology images. High bandwidth according to (J. G. Andrews, 2007; , Niyato et. al, 2007, Istepanian et. al, 2006, Zvikhachevskaya et. al, 2010) can as well help to support simultaneous transmission of various types of E-Health traffic. IEEE 802.16/WiMAX standard also allows application of encrypted functionalities via the MAC layer security features for healthcare data transmission.

One of the main issues related to the application of IEEE 802.16BWA (broadband wireless access) based technology for E-Health applications is service mapping. Recently, a number of publications have addressed this issue ( Istepanian et. al, 2006; Philip, 2008). Each of the proposed solutions has their own respective advantages and drawbacks. Although, there is a room for further optimization of this technique, the following mapping scheme is universally accepted for transferring E-Health data over WiMAX network (Philip, 2008):

- Allocate Unsolicited Grant Service (UGS) type of QoS to the biosignal traffic and voice conversation;

- $\quad$ Real-time Priority Service (rtPS) service for the video transmission;

- Non-real-time Priority Service (nrtPS) - to the file transfer, such as x-ray images and ultrasound results;

- Best Effort (BE) service class is to be allocated for the database access, e-mail exchange and web.

In the following research we utilize the above service mapping approach for the efficient EHealth related video streaming over IEEE 802.16 networks.

\section{B Distribution framework}

A novel concept is proposed to utilize object orientation of MPEG video streams for segmented distribution over IEEE 802.16 QoS-supported MAC infrastructure. We utilize a 
coded representation of media objects where each object is a part of complex audiovisual scene and can be perceived and processed separately. Most video distribution techniques aim at delivering MPEG streams with defined recommendation for protocol stack exploited within the communication procedures. QoS - supported network transmission technologies provide mechanisms for MPEG video distribution over its infrastructure inherently dictated by the dynamic nature of video traffic. In WiMAX networks service categories like rtPS, extended-real-time Priority Service (ertPS) and nrtPS are used for video-application data delivery depending on QoS needs for a certain video flow. Each Elementary Steam (ES) belonging to MPEG audiovisual flow can be characterized by stringent QoS requirements which are generally referred to one out of five service categories exploited in WiMAX.

Therefore, MPEG video can be transmitted through a defined MAC service connection of WiMAX system or, alternatively, many service connections of different service classes can be assigned to incoming MAC SDUs (service data units) of elementary streams segmented from the basic MPEG audiovisual scene.

The structural framework of traffic distribution in WiMAX simple topology is illustrated in Fig.1.3. In this figure, a Base Station (BS) is fully responsible for Up Link (UL) and Down Link (DL) traffic scheduling. Virtual UL scheduling process is integrated into the BS MAC architecture. The diagram schematically demonstrates data and signalling flows for UL communication between Service Station (SS) and BS. UL traffic from upper layer of MAC SDU units will be classified on the basis of QoS demands inherently allocated between

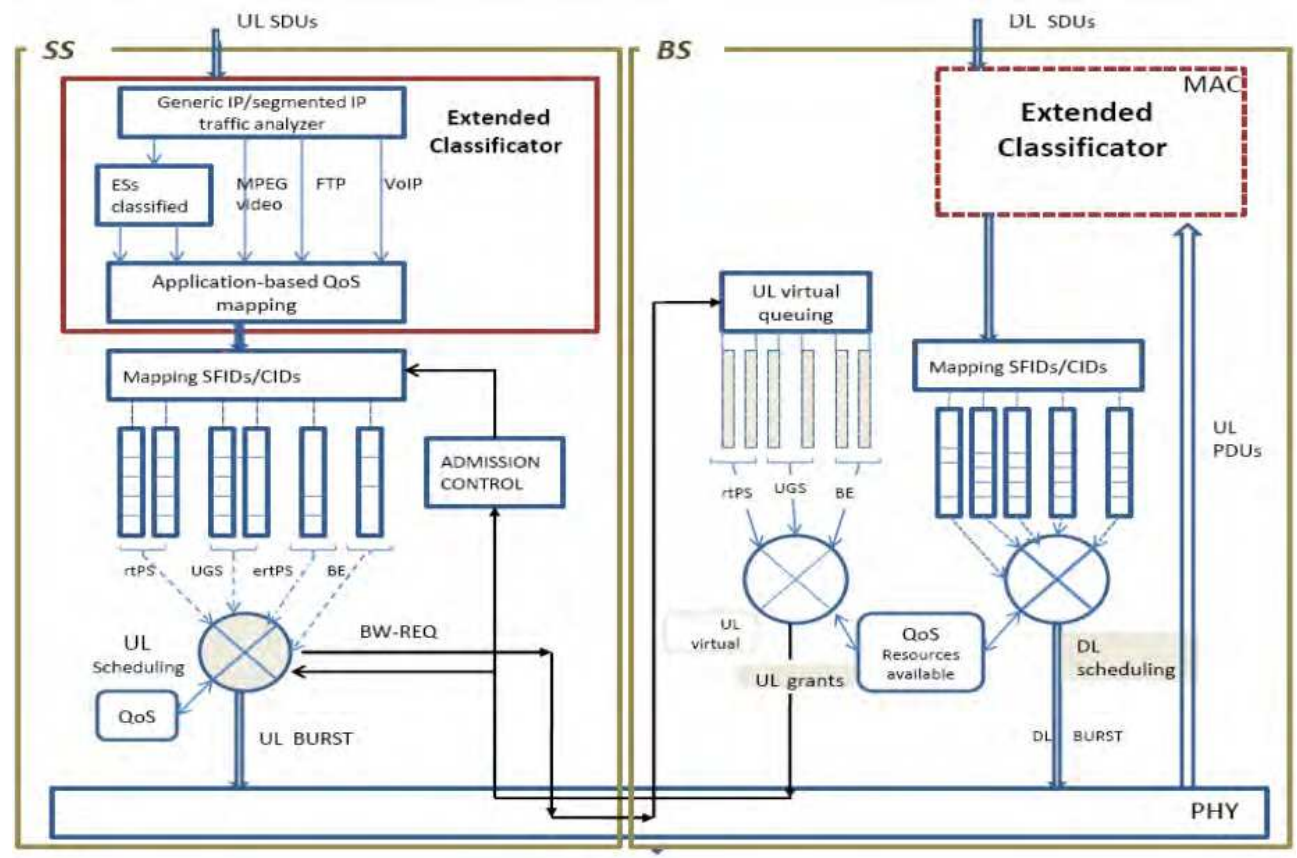

Fig. 1.3. Novel distribution framework to support object-based MPEG-4 video streams in WiMAX. 
already existed service connections or put in a buffer for further connection established in line with grant/rejection generated by a BS. In order to set up a new CID (connection identifier), initiated by incoming traffic, the Mobile Station (MS) utilizes a well-known handshaking procedure to request bandwidth resources from the BS (Andrews, 2007).With appearing needs of bandwidth increase for existing service connections due to the dynamic behaviour of real time video data, for example, it is the responsibility of the MS UL scheduler either to re-allocate available resources between established connections or address the BS for additional provisional QoS set. As shown in Fig.1.3, this request opportunity is realized by BW-REG signalling message, outgoing over the WiMAX control channel. When the BS grants the necessary bandwidth, the MS UL scheduler decides whether to delegate this allocation to the maintained CID connection or set up a new one. The scheduling policy and design are beyond of WiMAX standard scope and equipment vendors are encouraged for proprietary solutions, complied with general standard specifications ( Andrews, (2007).

Each service connection with packets waiting in the queue has a CID and service flow identifier (SFID) mapping to deliver packets with certain QoS guarantees to a destination address. The scheduling algorithm plays a major role in assigning burst profiles to awaited packets, and will be re-allocating the available resources, implement dropping and a connection admission policy corresponding to the distribution function and a mechanism presented in its design.

We extended the functionality of the conventional classifier/analyzer module integrated in WiMAX MAC layer to a number of specific tasks required to support the proposed algorithm. The upper SDU units will be analyzed with the purpose of determining IP packets belonging to segmented ESs or generic packets with MPEG payload. Furthermore, those from ESs are to be classified on the basis of QoS needs and then sent to the mapping block for correlating packets with QoS categories offered by WiMAX. Classified elementary streamed (ES) packets will be finally marked as application-based traffic in the category with similar QoS application needs. After that, the mapping module distributes traffic between unique service connections for supported QoS queues.

\section{Descripton of the Extended Clasificator}

In order to support the proposed modifications, we introduce Extended Classificator as shown in Fig. 1.3. The significant value of the integrated Extended Classificator is to simultaneously treat packets from both conventional MPEG-structured and segmented ES video streams to provides freedom to end-users for optional use of either one or another, or both, video transmission schemes. This separation could face a quality difference and be beneficially applied by service operators in commercial implementations.

For the purpose of data identification we introduce a traffic analyzer module, which is capable of determining incoming IP packets. These packets can belong to certain MPEGgeneric, MPEG segmented or conventional application payload types. In this architecture we add functions, such as handling and identification of MPEG and MPEG-ES related traffic. The classification of IP packets, such as from a Hypertext Transfer Protocol (HTP), Voice over IP (VoIP), and other services is specified by the standard. WiMAX MAC convergence sublayer is dedicated to manage the upper layer generated packets, as specified in packet header suppression (PHS) technology of the IEEE 802.16 standard (Andrews, 
2007). The proposed technique is fully compatible with the WiMAX specification and does not require any alterations in the standard. MPEG ES segmentation process is to be performed at upper layers. IP packets, incoming to WiMAX system elements, contain signalling information about its segmented parameters and initial audiovisual source.

In our previous research (Markarian et. al, 2010) we were focused on mapping ES packets to specific categories of traffic applications, such as MPEG-4 video, as presented in (Markarian et. al, 2010). The mapping rules proposed in this paper introduce modification for WiMAXenabled cross-layer data forwarding and are shown in Fig.1.4. In this diagram, each group of ES refers to a certain application type with the following classification for related IEEE802.16 service classes.

The header of the each layer bears significant information about associated links between ES data and further QoS treatment of incoming packets. We propose a cross-layer entity operating as a mapping/classification table to set up matching rules between communicating layers for delivering packets through the protocol suite. The operating layer is able to classify the incoming SDU by addressing to cross-layer table for inserting the correct information in the defined header field to inform lower layer of the requested services. However, the design and development of detailed protocol suite for ES-IP packet correlation mechanism and synchronization is beyond the scope of this paper and a topic of further detailed research. Meanwhile, it should be noted, that synchronization signalling data should be integrated into the single ES with premium QoS to provide guaranteed resources for delivery, as just the case with UGS service class.

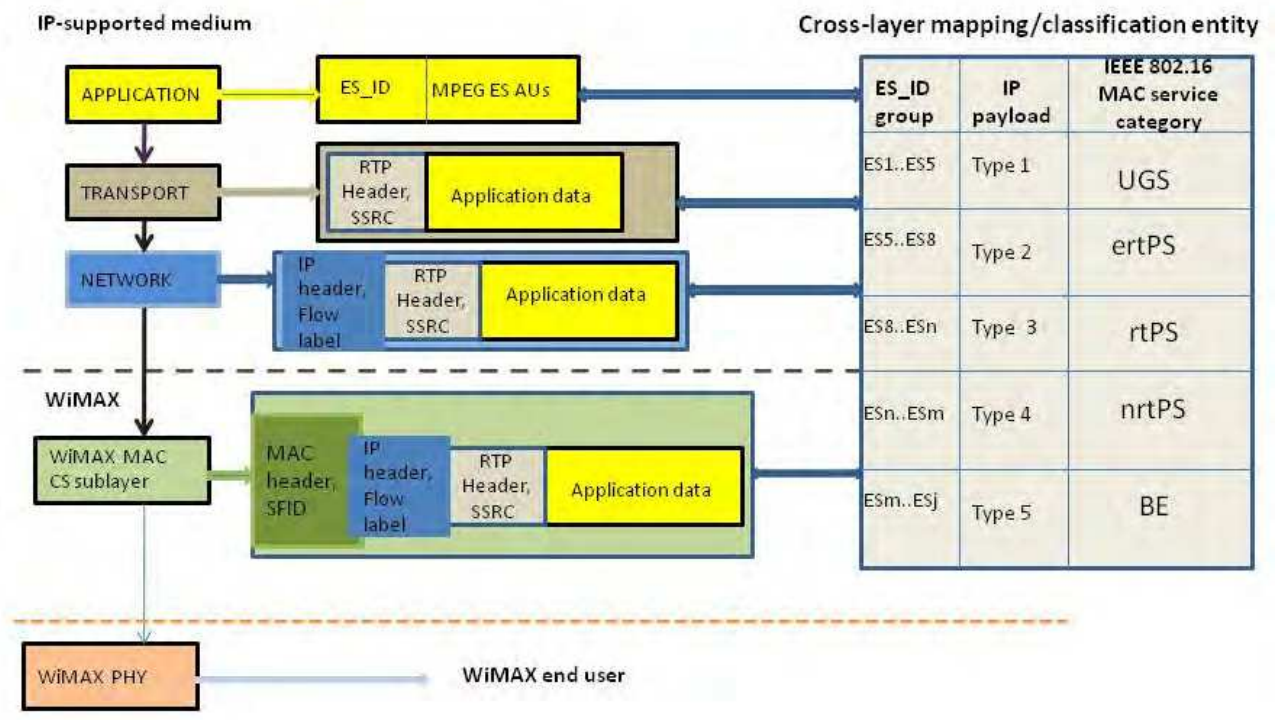

Fig. 1.4. Modified protocol-based cross-layer architecture.

One of the key aspects of video distribution over WiMAX is selecting of the right service class which will not be affected by the performance of the physical (PHY) layer. For 
example, Automatic Repeat Request (ARQ) of PHY layer dramatically improves the bit error ratio performance in pure (niseless) channel conditions. However, this mechanism introduces delays to the transmission of the video packets (Andrews, 2007; G.Markarian, 2010 a).

For our study we have chosen conventional Weighted Round Robin (WRR) algorithm for UL scheduling and developed a software model on the basis of the proposed program WiMAX module, elaborated for cooperative modelling within NS-2 simulator environment (Chen et. al, 2006). The results of this investigation could also be used in the future research related to optimum scheduling design.

\subsection{Simulation scenarios and experimental results}

\section{A Tests of a Novel Segmented Distribution Scheme with the Stress to E-Health Applications}

In the developed simulation model we implemented the direct functional correlation between the ESs and QoS scheduling categories offered in WiMAX. We assume that every ES with its QoS set can refer to a certain IEEE 802.16 MAC connection identified for the related service class UGS, rtPS, nrtPS, etc. which is associated with the specific healthcare application. Thus, this simulation approach means that the ES required for delivery of a data flow generated from a defined object with specific behaviour would get appropriate scheduling service as an individual stream with QoS-based application requirements.

\begin{tabular}{|c|l|c|c|}
\hline $\begin{array}{c}\text { Service } \\
\text { Class }\end{array}$ & \multicolumn{1}{|c|}{ Type of E-Health Transmitted Data } & $\begin{array}{c}\text { Packet } \\
\text { size, } \\
\text { Byte }\end{array}$ & $\begin{array}{c}\text { Data } \\
\text { rate, } \\
\text { Mbps }\end{array}$ \\
\hline UGS & Live Teleconference (video) & 200 & 2 \\
\hline rtPS & $\begin{array}{l}\text { Medical Video Transmission (surgery, tutorial, } \\
\text { presentation, video consultation) }\end{array}$ & 150 & 1 \\
\hline BE & Request to the Database & 40 & 0,02 \\
\hline
\end{tabular}

Table 1.2. Test Parameters For The First Scenario.

In the first scenario, which represents the conventional approach (Andrews, 2007), we establish three connections with different service classes, as indicated in Table 1.2. Fig. 1.5 illustrates simulation results for the conventional transmission of the first scenario, which is described in Table 1.2.

The next simulation set (as given in Table 1.3) presents simulation settings for the second scenario sets, where the developed technique is applied. The aim of this simulation is not only to test the technique but also to compare its performance over the conventional transmission scenario 1 and demonstrate advantages of the developed technique.

As shown in Table 1.3 both UGS and rtPS streams were split according to the proposed video distribution algorithm. For example, in scenario 2.1 of Table 1.3, the total UGS load of $2 \mathrm{Mbps}$ is divided into two UGS streams of $1 \mathrm{Mbps}$ each. Furthermore, the original $1 \mathrm{Mbps}$ connection referred to rtPS service is separated into two streams. These streams are ertPS and $\mathrm{BE}$ with data rates $0.6 \mathrm{Mbps}$ and $0.4 \mathrm{Mbps}$ respectively. In scenarios 2.2 and 2.3 (Table 1.3) the original UGS traffic rate is unchanged and the BE rate is constant through the whole simulation set. 


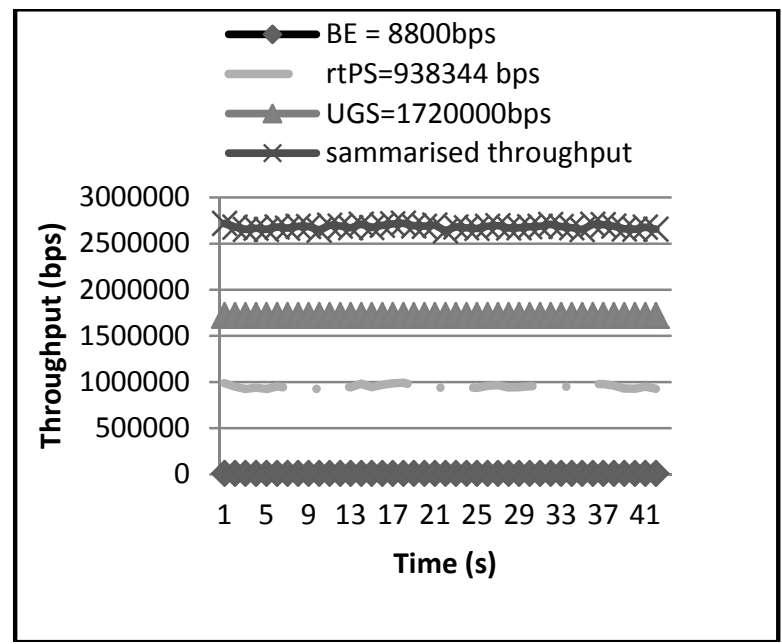

Fig. 1.5. Throughput comparison for the first (conventional) Scenario.

Fig. 1.6 shows comparative results in terms of summary throughput gain (system capacity gain), achieved for the second scenario in agreement with the parameters presented in Table 1.3.

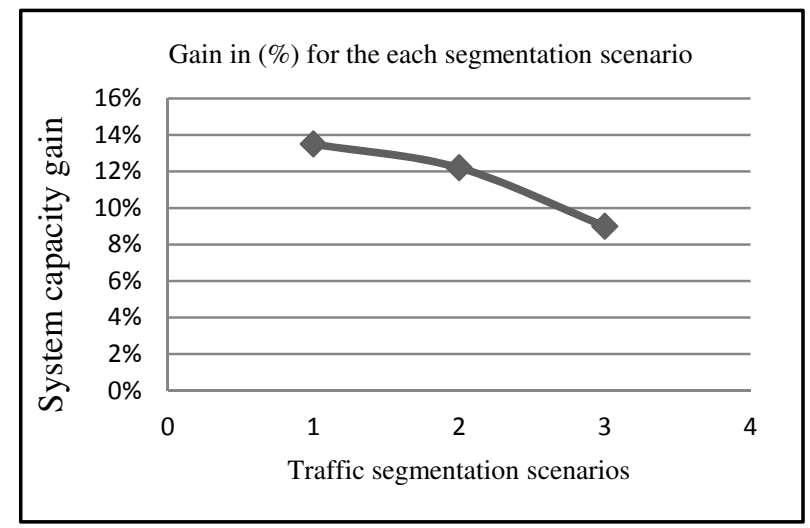

Fig. 1.6. System bandwidth gain for the Second Scenario.

The percentage gain is calculated on the basis of the comparison of the average summary throughput of conventional scenario with summary throughput results, obtained for the presented segmentation set scenarios:

$$
T=\frac{T_{\text {initial }}}{T_{\text {segmented }}},(\%)
$$

where Tinitial - is the summarised throughput for the initial video stream; Tsegmented - is the summarised throughput for segmented scenarios.

$$
\mathrm{T}_{\text {initial }}=\sum_{i=1}^{n} \mathrm{~T}_{\mathrm{i}}=\mathrm{T}_{\mathrm{UGS}}+\mathrm{T}_{\mathrm{rtPS}}+\mathrm{T}_{\mathrm{BE}} ;
$$


where $\mathrm{T}_{\mathrm{UGS}}, \mathrm{T}_{\mathrm{rtPS}}, \mathrm{T}_{\mathrm{BE}}-$ throughput results for UGS, rtPS and $\mathrm{BE}$ connections respectively.

$$
\mathrm{T}_{\text {segmented }}=\sum_{i=1}^{n} \sum_{\mathrm{k}=1}^{\mathrm{m}} \mathrm{T}_{\mathrm{ij}}
$$

where $i$ - number of service groups, $k$ - number of segmented streams within each service group.

\begin{tabular}{|c|c|c|c|c|c|c|c|c|}
\hline $\begin{array}{c}\text { Scenario } \\
\text { number }\end{array}$ & $\begin{array}{c}\text { UGS1, } \\
\text { load, } \\
\text { Mbps }\end{array}$ & $\begin{array}{c}\text { UGS2 } \\
\text { load, } \\
\text { Mbps }\end{array}$ & $\begin{array}{c}\text { ertPS } \\
\text { load, } \\
\text { Mbps }\end{array}$ & $\begin{array}{c}\text { rtPS } \\
\text { load, } \\
\text { Mbps }\end{array}$ & $\begin{array}{c}\text { BE1 } \\
\text { load, } \\
\text { Mbps }\end{array}$ & $\begin{array}{c}\text { BE2 } \\
\text { load, } \\
\text { Mbps }\end{array}$ & $\begin{array}{c}\text { Summary } \\
\text { load, } \\
\text { Mbps }\end{array}$ & $\begin{array}{c}\text { Total } \\
\text { bandwidth, } \\
\text { Mb }\end{array}$ \\
\hline № 2.1 & 1 & 1 & 0,6 & 0 & 0,4 & 0,02 & 3,02 & 3.5 \\
\hline № 2.2 & 2 & 0 & 0,4 & 0,5 & 0,1 & 0,02 & 3,02 & 3.5 \\
\hline № 2.3 & 2 & 0 & 0,3 & 0,5 & 0,2 & 0,02 & 3,02 & 3.5 \\
\hline
\end{tabular}

Table 1.3. Simulation Parameters for the Second Scenario Set

As illustrated in Fig. 1.6, the best gain ratio approximately $14 \%$ was obtained when most data are forwarded via connections that were served by rtPS and UGS services. In addition, this best indication is explained by exploiting of separation of the initial UGS stream of 2 Mbps load on two UGS connections accounted for $1 \mathrm{Mb}$ load per each.

This fact supports our assumption that the segmented approach would lead to better performance in the comparison with traditional IEEE 802.16 MAC delivery. Moreover, as expected, the WRR scheduler first serves packets with a higher priority service connection. Hence, the least successful indications with about $9 \%$ capacity gain are provided for the scenario № 2.3 .

Based on our evaluated results we conclude, that two sub-connected segmentation models might be a trade-off solution for delivery video data with 2-enchanced quality layers, with rtPS service reserved for E-health video conference transmission. Observing the performance of the described scenario, different video distribution models can be effectively exploited taking into account the scheduling design. Scheduling can evenly improve the performance, as our theoretical concept was experimentally approved with the simple WRR algorithm to which no specific properties were added for a selected service class-oriented priority provision.

The third scenario set is presented in Table 1.4. It is dedicated to study the variation in the overall network throughput when the segmentation scheme is applied. For example, in scenario 3.1 the initial $1 \mathrm{Mbps}$ rtPS stream was separated on $0.5 \mathrm{Mbps}$ rtPS, 0,4 ertPS and 0.1 $\mathrm{BE}$ connections; while in scenario 3.2 the same $1 \mathrm{Mbps}$ rtPS video was simulated as $0.1 \mathrm{Mbps}$ rtPS, 0.4 ertPS and $0.5 \mathrm{Mbps}$ BE separate streams. The throughput for the each connection was analyzed. System capacity gain results for the 3 set are presented in Fig.1.7. As it can be seen from this figure, the summarized throughputs for each splitting scheme (Table 1.4) are compared to the conventional simulation model which is presented in Table 1.2.

This proves our expectation that the variation of the video stream splitting has an impact on the overall system throughput. Knowing this fact, for each type of transmitted video (surgery, tutorial, presentation, video consultation, etc) it is possible to predict the 
throughput gain and hence envisage the gain for the other type of transmitted data: ertPS (VoIP) or/and BE (web, database access) services, as it was shown for the specific scenario.

\begin{tabular}{|c|c|c|c|c|c|c|c|}
\hline $\begin{array}{c}\text { Scenario } \\
\text { number }\end{array}$ & $\begin{array}{c}\text { UGS } \\
\text { load, } \\
\text { Mbps }\end{array}$ & $\begin{array}{c}\text { rtPS } \\
\text { load, } \\
\text { Mbps }\end{array}$ & $\begin{array}{c}\text { ertPS } \\
\text { load, } \\
\text { Mbps }\end{array}$ & $\begin{array}{c}\text { BE1 } \\
\text { load, } \\
\text { Mbps }\end{array}$ & $\begin{array}{c}\text { BE2 } \\
\text { load, } \\
\text { Mbps }\end{array}$ & $\begin{array}{c}\text { Summary } \\
\text { load, } \\
\text { Mbps }\end{array}$ & $\begin{array}{c}\text { Total } \\
\text { Bandwidth, } \\
\text { Mb }\end{array}$ \\
\hline № 3.1 & 2 & 0,5 & 0,4 & 0,1 & 0,02 & 3,02 & 3,5 \\
\hline № 3.2 & 2 & 0,25 & 0,25 & 0,25 & 0,02 & 3,02 & 3,5 \\
\hline № 3.3 & 2 & 0.1 & 0,4 & 0,5 & 0,02 & 3,02 & 3,5 \\
\hline
\end{tabular}

Table 1.4. Simulation Parameters for Third Scenario Set

Fig. 1.7 illustrates the gain in percentage among test sets in the third scenario. In this figure numbers 1, 2 and 3 of the traffic segmentation scenarios indicate scenarios 3.1, 3.2 and 3.3, respectively. The maximum bandwidth gain is obtained in the third set (scenario 3.3) and raise up to $16 \%$ above the conventional scenario.

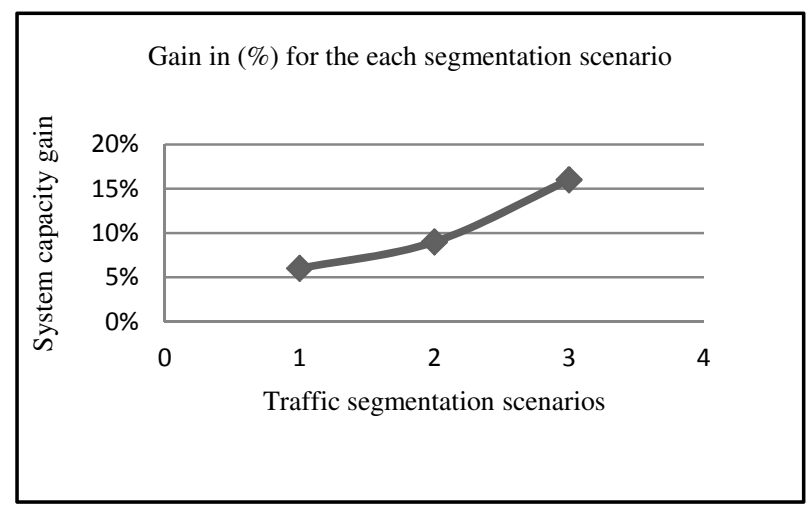

Fig. 1.7. System bandwidth gain for the third scenario.

It should be noted that we set the same values of the total system load and system bandwidth for all of the experiments. All the streams were re-allocated among the varied numbers of transport connections of defined QoS classes. It was made to model the variations of quality-selected video streams to compare network performance for the considered test scenarios. Our feasibility study demonstrates complete compatibility with the IEEE 802.16 standard.

\subsection{Results overview}

In this chapter section we described a novel video distribution approach designated for EHealth applications over IEEE 802.16 networks. The technique incorporates resource distribution, scheduling and content-aware video streaming taking advantage of a flexible QoS functionality offered by IEEE 802.16 technology. The proposed technique was thoroughly investigated under various scenarios, which included streaming video over MAC layer service connections. It is shown that the technique allows $9-16 \%$ increase in 
overall network bandwidth while maintaining full compatibility with IEEE802.16/WiMAX specifications. The exact gain is dependent upon initial system configuration and selection of WiMAX user parameters. In addition, simulation results shows that WiMAX-enabled Ehealth infrastructure is able to selectively handle numerous telemedicine application-driven traffic with required quality parameters within the available link budget.

\section{WiMAX-supported video distribution in surveillance applications}

\subsection{IEEE 802.16/WiMAX practical benefits in video surveillance}

Video surveillance technology has been exponentially increasing its presence among most public and private premises since its first introduction in the 1940s as a security tool for banking industry (Lalwani \& Kulasekare, 2011). Current demand for cost-effective and reliable video surveillance system is spread over most public places, like schools, universities, shopping malls, including specific security aspects, such as public transport and street traffic monitoring with aim of crime prevention and fast lawful response. To address the full range of technical issues associated with deployment, maintenance and target-oriented behavior, a contemporary video surveillance system has to employ mobility support, IP-complied platform, scalable and cost-effective installation. Since, there is multitude of high resolution video flows, simultaneously transported over any video security systems, an efficient QoS and resource allocation mechanisms are to be present in this system to optimally utilize available bandwidth.

Meanwhile, most IP wireless video surveillance systems adhere to WiFi/IEEE802.11 standard suite and therefore show essential drawbacks, related to limited distance performance of up to 100 meters. It also does not allow to operate across large areas and assumes indoor applications with inherently small outdoor surrounding coverage (Lalwani \& Kulasekare, 2011). In addition, WiFi is unable to provide strict security transmission standards and flexible QoS-based prioritized treatment of video flows what makes WiMAX/IEEE802.16 more favorable for video surveillance applications because of its PHY and MAC adopted properties.

Having designed, as a wireless backhaul of broadband data, WiMAX can efficiently manage video surveillance and adjacent voice, data traffic with deterministic QoS tool to ensure reliable and secure video transmission (Henshaw, 2008). Overlooking a deep insight into the standard, in overall, WiMAX-based video surveillance solution provides numerous valueadded features, in particular (Henshaw, 2008):

- Cost-economy and accessible system deployment (fiber trenching and optic adjustment efforts of similar wired network cost 5-10 times more than its WiMAX system equivalent implementations)

- Rapid deployment and system configuration ( as compared with fiber and copper wires WiMAX equipment can be virtually mounted anywhere and exploited under severe weather conditions with an ability of fast unit removing or location update, while making that economically inefficient for wired infrastructure. WiMAX system configuration can last up to a few hours when wired system installation requires months to accomplish preliminary trenching works.

- Flexibility and scalability. (Small and portable end-system WiMAX-enabled cameras are not permanently attached to a fixed location and can be removed to a new location. 
System expansion is not limited and can be easily upgraded with new subscriber units, quickly re-configured by a central BS/video-server terminal owing to flexible resource management facilities which contribute to the IEEE802.16 specifications.

- Reliable and secure communication (OFDMa-based transmission opportunities together with embedded error correction and packet restore mechanisms provide high security standards for video signal propagation)

- High system capacity ( Multi-stream video data from surveillance cameras strive for bandwidth-consuming and delay-sensitive QoS demands, whereas timely bandwidth fair allocation is of primary importance. MAC QoS support of WiMAX enables to handle multiple video traffic in case of gradual system extension and suits higher video quality needs if image resolution is varied upon request).

- Mobility and IP support. Mobility support in WiMAX enables to use surveillance equipment on public transport and transfer on-line video monitoring traffic to police vehicles. IP support makes the use of any WiMAX network segments feasible within any IP-compatible MAN or LAN infrastructure.

Research initiative on fruitful utilization of WiMAX technology for video surveillance applications has involved as the real test-bed investigation scenarios, so as computer modeling also. The chief commonplace of the conducted experiments is to demonstrate the suitable opportunities and practical benefits of the standard employment within the broad scope of specific video security applications.

In WEIRD project (Ciochina \& Condrachi, 2008) video surveillance application was integrated into the functional network operator, Romania Orange, with WiMAX technology used as a broadband access solution. The actual surveyed area embraces a local Buharest test-bed and some other test-beds performed in Portugal and Italy (Ciochina \& Condrachi, 2008). The key aspect of the test-bed scenarios was the use of actually working base-stations, engaged in service of the live ORANGE WiMAX network customers. Besides handling the traffic from real subscriber abonents, the base station manages video streams from the surveillance cameras installed across University campus. Throughout the experiments, video streams with different rates, resolution and quality were created and transported over WiMAX links with various QoS categories to elaborate a trade-off solution. The throughput, delay and jitter QoS metrics evaluated in course of multiple test scenarios show that WiMAX technology enables high quality video streaming for the set of video surveillance applications. The simulation provides more relevance to result analysis in terms of both research and business needs taking into consideration the real market performance environment.

Computer modeling provided in (Lalwani, S. Kulasekare, 2011) was aimed at estimation of WiMAX practicality for video surveillance application. QoS parameters like throughput, end-to-end delay, jitter and packet loss were selected for performance assessment basis and verified through sets of case-study scenarios with help of OPNET14 program simulation. Experimental scenarios diverge by number of users, its localization against the BS, various uplink coding and modulation schemes. The rtPS (real time polling service) service attribute was selected for video surveillance traffic, since this service class supports variable data-rate and packet-size parameters and is considered as a relevant category for video streaming by default in WiMAX recommendations. The conclusions presented in the manuscripts, prove to be theoretically-expected and define the backward correlation between the number of 
users, its distant localization from the Base Station and end to end delay. The higher order of modulation scheme results in packet loss increase as well as a longer distance between mobile nodes and the Base Station considerably affects uplink packet loss probability. Altogether, for all scenarios throughput and delay indications still remain within acceptable constraints, such as up to $5 \mathrm{Mbps}$ and less than $0.5 \mathrm{~ms}$, respectively, that is quite suitable for video surveillance application.

The issues of live video surveillance on public transport were investigated in (Ahmad \& Habibi, 2011). The real-time video communication from moving vehicles faces a significant technological challenge that is caused by multipath fading and consequent low throughput at high vehicle speeds due to technical constraints of the existing communication technologies. Despite the WiMAX/IEEE 802.16 ability to offer a guaranteed minimal date rate, it fails to cope with high packets error rate and maintain video traffic throughput sufficient for acceptable video quality in wireless mobile and speedy conditions. Due to ineffectiveness of lost packets retransmission recovery schemes, associated with considerable data overheads that get jitter and yet-low date rate application-unsuitable, error-control mechanisms, such as forward error control (FEC), are well-fitted for high speed wireless communication (Ahmad \& Habibi, 2011). These recovery mechanisms therefore have no corrupted packets retransmission involved. However, FEC schemes use variable number of parity bits, a FEC code size consists of. The FEC code size is completely relied on feedback data which bear actual information about current communication environment. In real mobile wireless conditions, fluctuating noise level creates untrue channel characteristics for adjusting an optimal FEC code size with resulting data missing or overhead. In (Ahmad \& Habibi, 2011) a novel FEC scheme was proposed to adaptively compute FEC code size in WiMAX video communications. The presented scheme is based on Reed-Solomon error correction code and includes 3 integral parts (Ahmad \& Habibi, 2011):

1. Assessment of bit error probability at different vehicular speeds in WiMAX

2. Utilization of these estimates for proactive adjusting FEC code size in live video communication.

3. Use of de-activation/offline camera mode when the WiMAX resources are considered to be insufficient for maintaining all video flows.

Simulation results, a computer performed, demonstrate that the proposed scheme makes WiMAX technology an efficient means for real-time video delivery at high vehicular speeds with the developed technique in use (Ahmad \& Habibi, 2011).

In the following we present and explore an efficient method for delivery of real-time video in multi-camera surveillance system which incorporates quality differentiation approach based on object tracking detection and QoS categorized policy brought forward by WiMAX technology. The aim of the conducted experiment is to verify the abilities of the proposed scheme to ensure more efficient management of WIMAX-based network capacity. Issues of optimal utilization of the saved bandwidth for transmission of additional traffic from active surveillance system elements were as well under exploration through NS-2 software computer simulation.

In (Tsitserov et. al, 2008) MPEG-based video distribution of object-oriented elementary streams over IEEE 802.16 networks is proposed. Further on, we expand this technique and 
suggest a selective quality control of the outgoing video streams, depending on a nature of objects detected or identified within a span. With the WiMAX flexible tools involving adjustment of service parameters for data transportation, we can ensure system resources for superior (high definition) HD video, whereas, (standard definition) SD or (low definition) LD video traffic will be assigned to available bandwidth in accordance with defined priorities. Such a control of the video quality allows solving the key surveillance challenge : detailed identification of a selected object for further recording and later analysis. Thus, dynamic allocation of available bandwidth in accordance with the proposed criteria enables optimization of system bandwidth. The simulation results show that the proposed technique is able to enlarge the covered surveillance area at the expense of saved bandwidth or allocate the released resources for additional data distribution upon selected case-study scenarios.

The rest of this sub-chapter is organized as follows: in the next section the proposed solution is described in details. In section 2.3 we present case-study scenarios together with simulation parameters. Experimental results analysis is given in section 2.4, and finally, in section 2.5, conclusions are provided for consideration and further research potential.

\subsection{The basic attributes of the verified method}

For the purpose of optimal utilization of the available system budget, we admit that dynamic regulation of outgoing video traffic will totally result in economy of bandwidth consumed and enable for extension of the surveillance area coverage. In most scenarios superior video quality allows detecting criminal identity, or details of negative factors. In conventional monitoring and surveillance systems, motion detection combined with object detection sensors are used for activation of monitoring functions of video cameras (Emilio Maggio \& Andrea Cavallaro, 2011). The schematic illustration of the WiMAX-based surveillance network is presented in the Fig.2.1. Each camera has an embedded motion sensor (or any alarm event sensor) to react to some supposed actions within viewing ability of a particular camera. As depicted on this Figure, №1, №2 and №3 cameras keep following a moving object until it is tracked by №4 and №5 cameras. According to our approach, HD video is transmitted from first 3 cameras, but №4 and №5 deliver low or standard definition video. Once image is caught by cameras № 4 and № 5, these cameras will switch to HD while first 3 cameras will turn back to SD.

All video flows are received by the BS and then transported globally to the monitoring center for recording and archiving. Moreover, mobile or fast response teams are aware of the controlled sector in case of the total surveillance area is divided between fast response groups.

The BS will multicast total traffic to all groups or forward specific video streams to a dedicated user. To realize that, the BS should involve an Operation Server for video stream processing and re-distribution of data flow upon user request. In our solution, we also provide various QoS boundaries for quality segmented video flows based on service class categories, introduced in WiMAX. Therefore, HD streams will be given higher priority and served first as UGS data, then SD video corresponds to rtPS class, and LD flows are classified as BE data and served in the last turn together with additional control data. Therefore, the best service and most resources are allocated to streams with HD quality to 


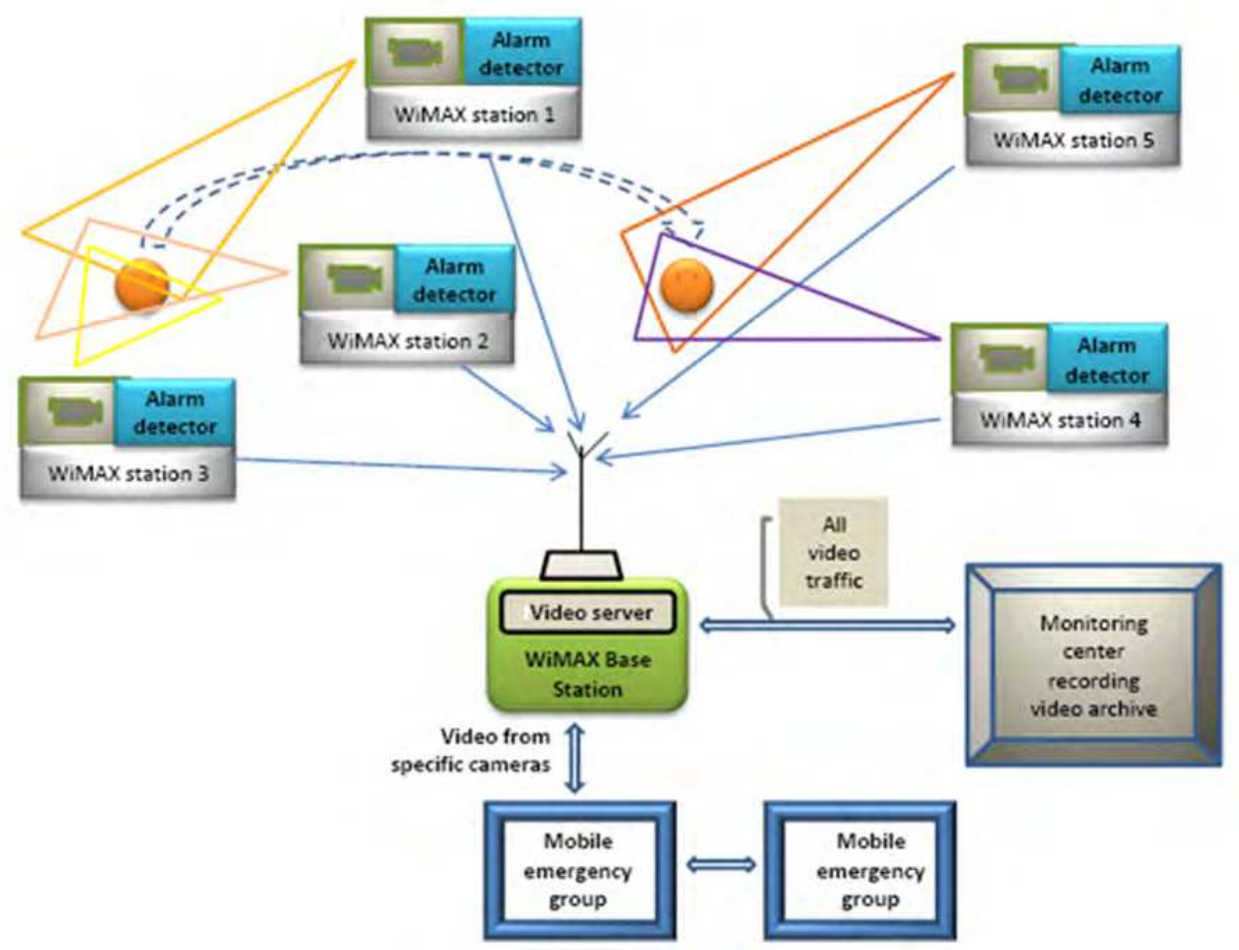

Fig. 2.1. WiMAX-based video surveillance system.

support high throughput for intensive traffic, but the rest of the bandwidth is delegated to video flows with less stringent boundaries for latency and throughput. In case of data drops or video artifacts in SD and LD video, most important information will be reflected in HD, triggered by event alarm, and can be re-produced with upper quality at the expense of better service treatment of HD video data. With introduction of categorized treatment of quality-selected video flows the surveillance network can dynamically re-allocate WiMAX resources between stations with cameras in such a way, that the whole controlled sector will be constantly covered and monitored, whereas any suspicious event is to be immediately fixed and recorded with a high resolution at premium quality. In comparison with a frequently-used video monitoring and wireless transport technology, like WiFi (IEEE 802.11) or direct PMP (point-to-multipoint) digital communication, no service guarantee for HD data can be provided, so all the streams are serviced equally or with a contention-based policy. That inevitably affects the video quality of HD video what results in failure to accomplish identification to a required extent.

We also show that some additional controlling information like GPS location of the object or camera map location can be easily transmitted together with video data, since WiMAX BS can delegate the rest of available bandwidth for such data communication as a BE service with no guarantee for latency and rate. These data can be transmitted during detection gaps, when most cameras are in state of LD video distribution. 


\subsection{Case study scenarios}

In our simulation we first assume baseline scenario, when network topology involves 2 cameras with HD video streams and 1 additional flow served for delivery of SD data but with less important service requirements. Both $\mathrm{HD}$ flows are set with $8 \mathrm{Mbsec}$ data rate and treated as UGS connections with ensured bandwidth allocation. This scenario describes the situation when the standard approach is applied and the total video is received with superior quality from 2 cameras and standard quality with $4 \mathrm{Mbsec}$ rate from 1camera for surveillance purpose. The total system load consists of video streams produced by installed cameras. Network bandwidth is constant and well sufficient for effective management of the incoming traffic for all scenarios.

For the second test we left the same full system load and bandwidth parameters, but apply the proposed technology and add more connections accounted to increased number of cameras. UGS connection with $8 \mathrm{Mbpsec}$ corresponds to the camera with HD video, but 3 rtPS connections with $4 \mathrm{Mbs}$ load each referred to 3 cameras enabling SD video transmission and imply no event details are tracked within their viewing sector.

\begin{tabular}{|c|c|c|c|c|c|c|c|c|}
\hline $\begin{array}{l}\text { Test } \\
\text { №1 }\end{array}$ & $\begin{array}{l}\text { Rate, } \\
\text { Mbsec }\end{array}$ & $\begin{array}{l}\text { Total } \\
\text { Data, } \\
\text { MB }\end{array}$ & $\begin{array}{l}\text { Total } \\
\text { system } \\
\text { bandwidth, } \\
\text { MB }\end{array}$ & \begin{tabular}{|l|} 
Simulation \\
time, \\
sec
\end{tabular} & Transmission & $\begin{array}{l}\text { Channel } \\
\text { Bandwidth, } \\
\mathrm{MHz}\end{array}$ & $\begin{array}{l}\text { PHY } \\
\text { mode }\end{array}$ & $\begin{array}{l}\text { Number } \\
\text { of MSs }\end{array}$ \\
\hline UGS1 & 8 & \multirow[t]{3}{*}{20} & \multirow[t]{3}{*}{28} & \multirow[t]{3}{*}{6} & \multirow[t]{3}{*}{ DL } & \multirow[t]{3}{*}{5} & \multirow{3}{*}{$\begin{array}{l}512 \\
\text { S- } \\
\text { OFD } \\
\text { MA }\end{array}$} & 1 \\
\hline UGS2 & 8 & & & & & & & 1 \\
\hline rtPS1 & 4 & & & & & & & 1 \\
\hline \multicolumn{9}{|c|}{ Test №2 } \\
\hline UGS1 & 8 & \multirow[t]{4}{*}{20} & \multirow[t]{4}{*}{28} & \multirow[t]{4}{*}{6} & \multirow[t]{4}{*}{$\mathrm{DL}$} & \multirow[t]{4}{*}{5} & \multirow{4}{*}{$\begin{array}{l}512 \\
\text { S- } \\
\text { OFD } \\
\text { MA }\end{array}$} & 1 \\
\hline rtPS1 & 4 & & & & & & & 1 \\
\hline rtPS2 & 4 & & & & & & & 1 \\
\hline rtPS3 & 4 & & & & & & & 1 \\
\hline \multicolumn{9}{|c|}{ Test №3 } \\
\hline UGS1 & 8 & \multirow[t]{7}{*}{20.2} & \multirow[t]{7}{*}{28} & \multirow[t]{7}{*}{6} & \multirow[t]{7}{*}{ DL } & \multirow[t]{7}{*}{5} & \multirow{7}{*}{$\begin{array}{l}512 \\
\text { S- } \\
\text { OFD } \\
\text { MA }\end{array}$} & 1 \\
\hline rtPS1 & 3 & & & & & & & 1 \\
\hline rtPS2 & 3 & & & & & & & 1 \\
\hline rtPS3 & 3 & & & & & & & 1 \\
\hline BE1 & 1.5 & & & & & & & 1 \\
\hline BE2 & 1.5 & & & & & & & 1 \\
\hline BE3 & 0.2 & & & & & & & 1 \\
\hline
\end{tabular}

Table 2.1. Simulation parameters. 
Finally the $3^{\text {rd }}$ test embraces the same full load segmentation principles, as exemplified in the second test, but we reduce traffic on rtPS connections down to $3 \mathrm{Mbsec}$, while 2 more BE connections with $1.5 \mathrm{Mb} / \mathrm{sec}$ load were set to simulate cameras with LD video flows. In this test we add new BE connection with $0.2 \mathrm{Mbsec}$ load to imitate control data transmission, such as GPS location or image delivery. Thus, in the last scenario we admit that $3 \mathrm{rtPS}$ cameras were switched to less consuming mode with rare frame/second rate video, or black/white color transmission, but the rest of the total system load was allocated for new 2 cameras with LD video traffic transported over BE connections respectively. Moreover, an additional $0.2 \mathrm{Mbs} \mathrm{BE}$ connection produces a slight increase in the total system load for adequate analysis. The main simulation parameters of the considered tests are provided in the Table 2.1.

It should be noted that we set the same values of the total system load and system bandwidth for most of the experiments, except the final scenario with a small load overcome. The total data amount is re-allocated between the varied number of transport connections of defined QoS classes to model the variations of quality-selected video streams to compare network performance for the considered test scenarios. Summary throughput comparison is illustrated in Figure 1.5. Every graph on this figure correlates to summarized throughput values of a particular test.

The whole simulation was carried out with support of WiMAX software module for NS-2 simulator designed by Chen, Wang, Tsai and Chang and proposed in (Chen et. al, 2006).

\subsection{Simulation results analysis}

With much attention to HD video streams we should note that the higher date rate of about $7 \mathrm{Mbsec}$ for UGS connection corresponding to superior video transmission, levels out around the same value throughout the whole experiment. This fact intensely shows that for all cameras with higher level of QoS requirements, WiMAX provides with sufficient resources to deliver superior video in spite of a number of supplementary cameras generating traffic with lower QoS needs. This is explained by QoS scheduling policy in which UGS connections are given priority amid the rest and the required resources are first delegated to serve these traffic delivery. Thus, the experimental figures demonstrate that the most important video with HD selected quality is supplied at the requested level.

With gradual network expansion, the system is again capable of providing distribution with support of required QoS metrics for both UGS and rtPS connections, as exemplified in Figure 2.3. rtPS connections with date rates surrounding default parameters of $4 \mathrm{Mbs}$ and $3 \mathrm{Mbs}$ are illustrated in Figures 2.2, 2.3 and 2.4 respectively. Thus, the system is flexible to optimize available bandwidth in a way, when service needs for traffic with HD and SD level are properly satisfied. The similar tendency was revealed in (Markarian et. al, 2010).

In the final Test 3 the system extension to 3 new cameras have led to $40 \%$ drop in rate values for BE connections, as described in Figure 2.4. To sustain data rates steady for connections of higher service categories, the system is slower to serve BE. Besides, no service guarantee is provided for BE connections and, therefore, exemplified as lower experimental indications in comparison with required ones. 


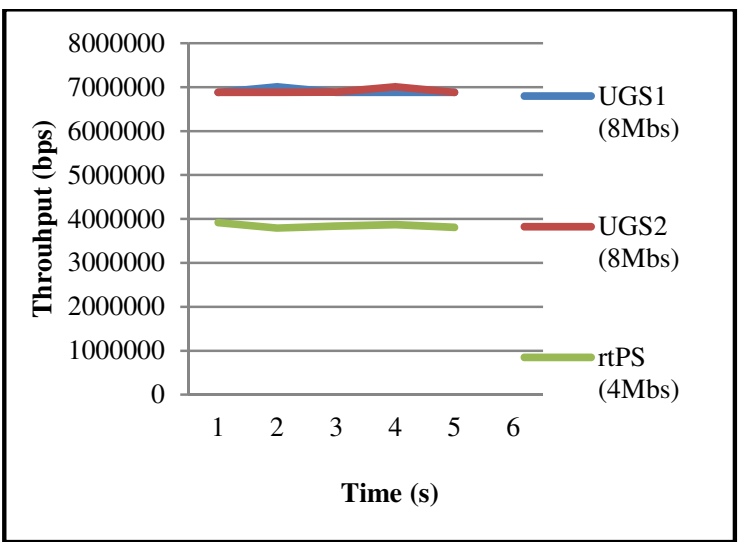

Fig. 2.2. Throughput results for Test 1.

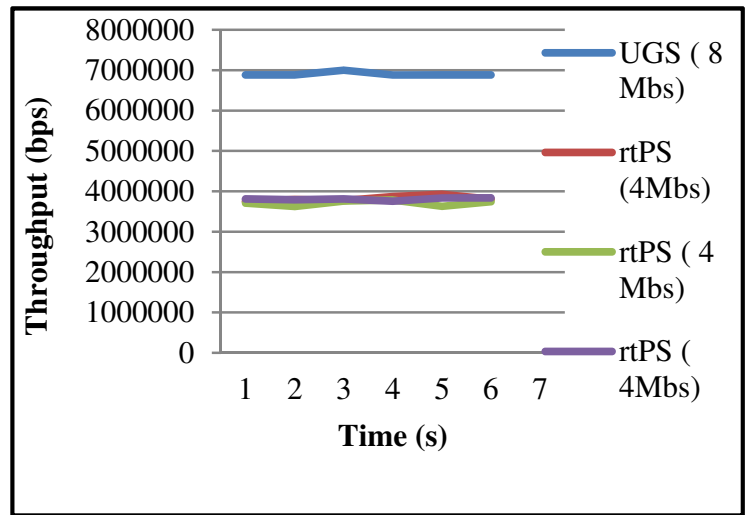

Fig. 2.3. Throughput results for Test 2.

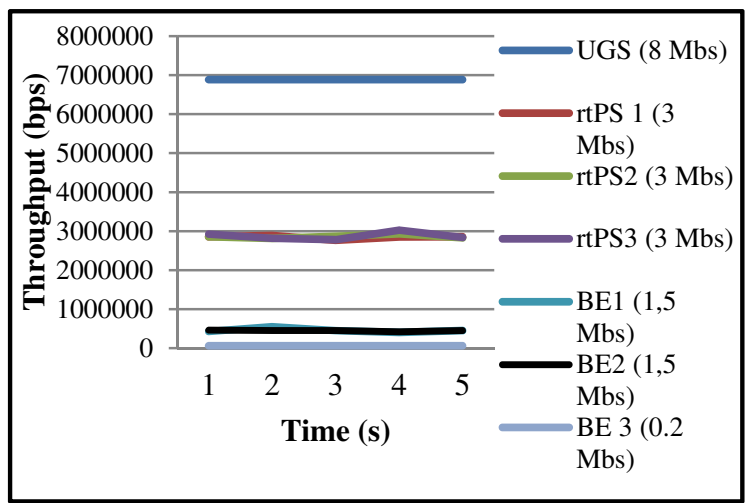

Fig. 2.4. Throughput indications for Test 3 


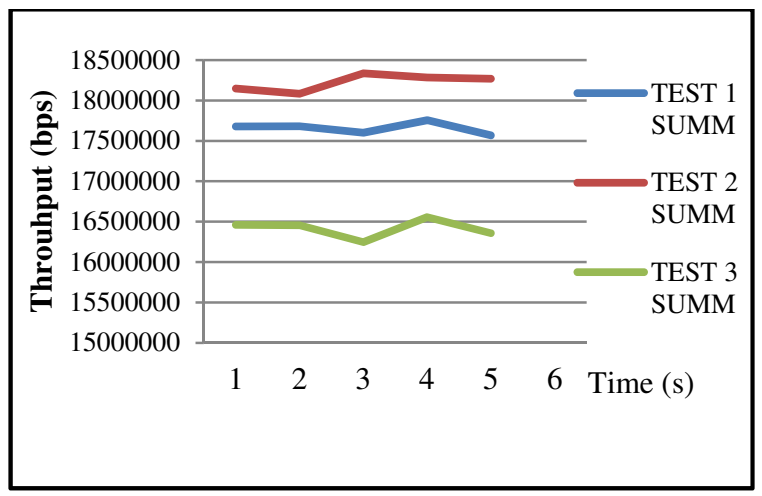

Fig. 2.5. Summary throughput comparison.

Nevertheless, with implementation to a real-life scenario, cameras with LD streaming transmit less timely-important information, therefore, the prioritized video uses UGS-based connection. Thus, lower data rate and higher delay are still justified by our introduced concept for selective video-quality in surveillance applications. Each time an alarm situation is detected, superior video quality is delivered along with rare frame/second rate video from LD network cameras enabling to properly react to emergency event and control the environment simultaneously. Based on summary throughput analysis, depicted in Figure 2.5, we observe that the lower value of around $16 \mathrm{Mbsec}$ was obtained for the most complicated network topology comprising of 7 terminals. This throughput indication is 17 $\%$ less than maximum figure of $18.3 \mathrm{Mbsec}$ achieved in Test 2 with only HD and SD traffic involved.

The minimal value of summary throughput, demonstrated in the Test 3 , is a result of smaller resources allocated for BE connections with data rates well below default figures. In this case, the system provides low date rate to save additional bandwidth, as BE data can be delivered within longer period with higher latency, hence summary throughput dropped, illustrating $17 \%$ bandwidth economy in comparison with an indication of Test 2.

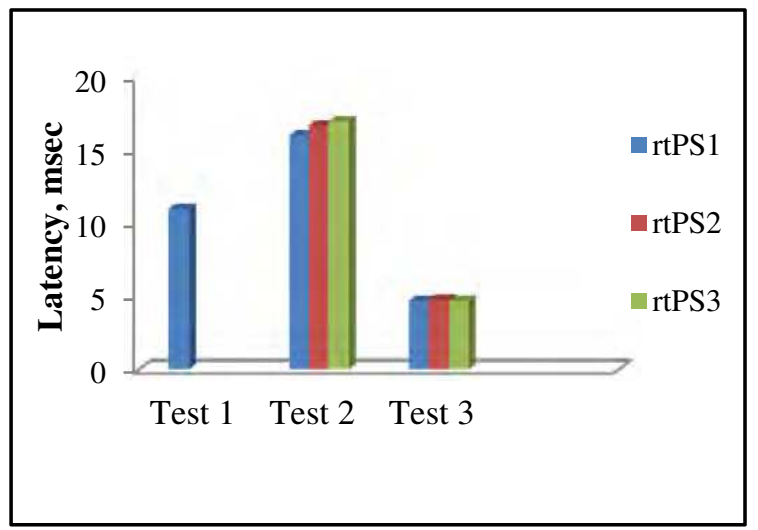

Fig. 2.6. Average latency for rtPS traffic. 
Average latency values, depicted in Figure 2.6 for rtPS connections, demonstrate that the minimal figures were obtained for Test 3 , in which the system resources were utilized in the best way, thanking to allocation of some of the total load for delay-tolerant BE connections of LD video and image/data traffic.

\subsection{Simulation outcome}

In this section we introduce an efficient distribution technique for multiple video streams over WiMAX-based monitoring and surveillance networks. We performed a computer simulation of the selected case-study scenarios which incorporate dynamic quality-based adaptation of video data entering the system and QoS categorized support for incoming traffic with HD, SD and LD quality.

The experimental results demonstrate that the introduced concept enables an optimized system resource utilization in case of network extension within the constant system bandwidth. The test results proves the feasibility of supplementary control data distribution with no service guarantee together with important HD video streams when the system is managed with help of video quality selection with integrated alarm-driven functionality.

The fulfilled experimement opens ways to theoretical foundation for successful implementation of QoS-supported $4 \mathrm{G}$ systems in surveillance application with trafficconsumed real-time video delivery.

\section{Conclusions}

In the provided chapter we have described an efficient methodology to support real-time video delivery in E-health and video surveillance applications over WiMAX systems. We have experimentally shown how WiMAX technology is able to satisfy stringent demands for bandwidth-consuming and delay-sensitive video traffic distribution in specified application areas. In overall, the developed technique demonstrates considearble achievments in system bandwidth optimization and ensures the reliable system performance under the selected cased-study scenarios. The proposed technique also reflects flexibility of the WiMAX QoSsupported concept in order to be successfully exploited for real-time video transmission across telemedicine and video surveillance multi-user networks.

\section{Acknowledgement}

This work was supported by the EU FP7 WiMAGIC Project and authors would like to express their gratitude to Rinicom Ltd for the opportunity to work on this project.

\section{References}

Article is available from encyclopedia Wikipedia, "eHealth". Wikipedia the free encyclopaedia. [Online] Available from:

http:/ /en.wikipedia.org/wiki/EHealth [Accessed: 5 March, 2010]

A.K. Zvikhachevskaya (2010). “Novel Wireless Communication Systems and Protocols for E-health Applications", PhD thesis, available at the Lancaster University PhD database, Lancaster, UK 
H. A. Skinner, O. Maley, C. D. Norman (2006). “Developing Internet-Based eHealth Promotion Programs: The Spiral Technology Action Research (STAR) Model". Health Promot Pract 2006; 7; 406 originally published online Jul 13, 2006; DOI: 10.1177/1524839905278889. [Online]. Available from: http://hpp.sagepub.com/ cgi/content/abstract/7/4/406]

Bobadilla, P. Gomez, J. I. Godino, Mapaci (2007). A Real Time e-Health Application to Assist Throat Complaint Patients", iciw, pp.63, Second International Conference on Internet and Web Applications and Services (ICIW'07)

F. Hu, S. Kumar. (2003). "QoS Considerations in Wireless Sensor Networks for Telemedicine". Proceedings of SPIE ITCOM Conference, Orlando, FL, 2003.

Y. Lin, et al., (2004). "A Wireless PDA Based Physiological Monitoring System for Patient Transport". IEEE Transactions on Information Technology in Biomedicine, 2004, Vol. 8, issue 4, p. 439-447. [Online]. Available from:

http://www.ncbi.nlm.nih.gov/ pubmed/15615034 [Accessed: September 2009].

F. Hu, S.Kumar, (2006). "The Integration of Ad hoc sensor networks and Cellular Networks for Multi-class Data Transmission". Ad hoc Networks Journal (Elsevier), 2006. Volume 4(Issue 2): p. 254-282. ISSN:1570-8705. [Online]. Available:

http:/ / portal.acm.org/citation.cfm?id=1640928 [Accessed: September 2008].

N. Maglaveras, et al., (2002). "Home care delivery through the mobile telecommunications platform: the Citizen Health System (CHS) perspective". International Journal of Medical Informatics, 2002. Vol. 68: p. 99-111. [Online]. Available:

http:/ /linkinghub.elsevier.com/retrieve/pii/S1386505602000692[Accessed:Septem ber 2008].

P.Dudzik, et al., (2009). "Wireless ATM as a base for medical multimedia applications and telemedicine". Computer Systems and Applications - CSA'98, Irbid, Jordan. April 1998. [Online]. Available:

http://en.scientificcommons.org/43302879 [Accessed: September 2009].

D. J. Vergados, D. D. Vergados., I. Maglogiannis, (2006). “Applying Wireless DiffServ for QoS Provisioning in Mobile Emergency Telemedicine". IEEE Communications Society subject matter experts for publication in the IEEE GLOBECOM 2006 proceedings, 2006.

Y. Liu, et al., (2006). “Dynamic Admission and Congestion Control for Real-time Traffic in IEEE 802.11e Wireless LANs". 2006 IEEE International Conference on Wireless and Mobile Computing, Networking and Communications, ISBN: 1-4244-0494-0, June 19June 21, 2006. [Online] Available:

http://www.computer.org/portal/web/csdl/ doi/10.1109/WIMOB.2006.1696391 [Accessed: March 2009].

D. Gao, J. Cai, (2005). “Admission Control with Physical rate measurement for IEEE 802.11e Controlled Channel Access".IEEECommunicationLetters,vol.9,no.8,August2005. [Online]Availilable:

http://ant.comm.ccu.edu.tw/course/94_WLAN/1_Papers/LTR,\%20Admission \% 2 0control\%20with\%20physical\%20rate\%20measurement\%20for\%20IEEE\%20802.11e $\%$ 20controlled \%20channel\%20access.pdf

K. Noimanee, S. Noimanee, P. Khunja, and P. Keawfoonrungsie, (2010) “Medical Consultbased System for Diagnosis on WiMAX Technology", International Journal on Applied Biomedical Engineering Vol. 3, No. 1, p.51-55, 2010. 
D.Tsitserov, G. Markarian, I. Manuilov, (2008) “Real-Time Video Distribution over WiMAX Networks", Proceedings of the 9th Annual Postgraduate Symposium "The Convergence of Telecommunications, Networking and Broadcasting", PGnet Conference, Liverpool, 23-24 July, 2008. Available from:

http://www.cms.livjm.ac.uk/pgnet2008/Proceeedings/Papers/2008019.pdf. (URL).

G. Markarian, D. Tsitserov, A. Zvikhachevskaya, (2010). “Novel Technique for Efficient Video Distribution over WiMAX networks". Proceedings of 21st Annual IEEE International Symposium on Personal, Indoor and Mobile Radio Communications (PIMRC 2010) , 2010, Istanbul.

J. G. Andrews, (2007). "Fundamentals of WiMAX: Understanding Broadband Wireless Networking", in Prentice Hall Communications Engineering and Emerging Technologies Series. Prentice Hall, 2007.

D.Niyato, E.Hossain, J.Diamond, (2007). “IEEE 802.16/WiMAX-Based Broadband Wireless Access and Its Application for Telemedicine/e-Health Services". IEEE Wireless Communications, p. 1536-1284. February 2007.

D. Tsitserov, A. Zvikhachevskaya, (2010). "The Novel Cross-Layer Algorithm for Distribution of MPEG-4 ES-segmented Flows over IEEE 802.16". Proceedings of $4^{\text {th }}$ International Symposium on Broadband Communications, ISBC2010. Malaysia. 2010.

R. S. H. Istepanian, S. Laxminarayn, C. S Pattichis, (2006). "M-Health: Emerging Mobile Health Systems", Springer-Verlag, ISBN/ISSN 0387265589, London.

A. Zvikhachevskaya, G.Markarian, L. Mihaylova, (2009). “QoS consideration for wireless telemedicine/e-health services". Proceedings of the IEEE Wireless Communications and Networking Conference WCNC, Budapest, Hungary, 2009.

N. Y. Philip, (2008). "Medical Quality of Service for Optomised Ultrasound Streaming in Wireless Robotic Tele-Ultrasonography System ", PhD Thesis, Kingston University. 2008.

«Overview of the MPEG-4 Standard», (2002). ISO/IEC JTC1/SC29/WG11, N4668, March 2002.

G.Markarian, (2010 a). "Wireless Broadband Communications for Video Surveillance Trends, Problems and Solutions" (invited tutorial). Proceedings of the $13^{\text {th }}$ International simposium on Wireless Personal Multimedia Communications, p.13. October 11-14, Recife, Brazil. 2010.

J. Chen , C.-C. Wang, F. C.-Da Tsai, C.-Wei Chang, (2006). “The Design and Implementation of WiMAX Module for ns-2 Simulator", Proceedings of the WNS2'06 conference, October 10, 2006, Pisa, Italy.

M. Lalwani, S. Kulasekare, (2011).“Analysis of Video Surveillance over WiMAX Networks”, Final Report, Simon Fraser University, April, 2011; available from: www.sfu.ca/-mla17/ENSC427.HTML

R. Henshaw, (2008). “The Wireless Video Surveillance Opportunity: Why WiMAX is not just for Broadband Wireless Access", WiMax.com Broadband Solutions, Inc.,2008; available from:

http:/ / www.wimax.com/features/the-wireless-video-surveillance-opportunitywhy-wimax-is-not-just-for-broadband-wireless-access. 
D. S. Ciochina, C.A. Condrachi, (2008). “Video Surbeillance Application Using WiMAX as a Wireless Technology", Orange Romania/Technical Department, 2008; available from: www.orange.ro.

I. Ahmad, D. Habibi, (2011). "A WiMAX Solution for Real-Time Video Surveillance in Public Transport", International Journal of Computer Networks \& Communications (IJCNC) Vol.3, No.2, March 2011.

Dr. Emilio Maggio, Dr. Andrea Cavallaro. (2011). "Video Tracking: Theory and Practice", «Wiley», 292p., January 2011. 


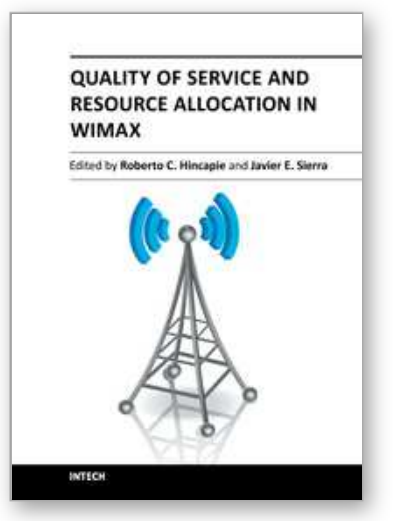

\author{
Quality of Service and Resource Allocation in WiMAX \\ Edited by Dr. Roberto Hincapie
}

ISBN 978-953-307-956-1

Hard cover, 376 pages

Publisher InTech

Published online 03, February, 2012

Published in print edition February, 2012

This book has been prepared to present state of the art on WiMAX Technology. It has been constructed with the support of many researchers around the world, working on resource allocation, quality of service and WiMAX applications. Such many different works on WiMAX, show the great worldwide importance of WiMAX as a wireless broadband access technology. This book is intended for readers interested in resource allocation and quality of service in wireless environments, which is known to be a complex problem. All chapters include both theoretical and technical information, which provides an in depth review of the most recent advances in the field for engineers and researchers, and other readers interested in WiMAX.

\title{
How to reference
}

In order to correctly reference this scholarly work, feel free to copy and paste the following:

Dmitry V. Tsitserov and Dmitry K. Zvikhachevsky (2012). Efficient Video Distribution over WiMAX-Enabled Networks for Healthcare and Video Surveillance Applications, Quality of Service and Resource Allocation in WiMAX, Dr. Roberto Hincapie (Ed.), ISBN: 978-953-307-956-1, InTech, Available from:

http://www.intechopen.com/books/quality-of-service-and-resource-allocation-in-wimax/efficient-videodistribution-over-wimax-enabled-networks-for-healthcare-and-video-surveillance-appli

\section{INTECH}

open science | open minds

\section{InTech Europe}

University Campus STeP Ri

Slavka Krautzeka 83/A

51000 Rijeka, Croatia

Phone: +385 (51) 770447

Fax: +385 (51) 686166

www.intechopen.com

\section{InTech China}

Unit 405, Office Block, Hotel Equatorial Shanghai

No.65, Yan An Road (West), Shanghai, 200040, China

中国上海市延安西路65号上海国际贵都大饭店办公楼 405 单元

Phone: +86-21-62489820

Fax: $+86-21-62489821$ 
(C) 2012 The Author(s). Licensee IntechOpen. This is an open access article distributed under the terms of the Creative Commons Attribution 3.0 License, which permits unrestricted use, distribution, and reproduction in any medium, provided the original work is properly cited. 